\title{
Limitations of a Relaxation Oscillator in Capacitance Measurements
}

\author{
Yili Liu, Song Chen, Masakatsu Nakayama, and Kenzo Watanabe, Fellow, IEEE
}

\begin{abstract}
Oscillation periods of a relaxation oscillator consisting of an op-amp-based integrator and a comparator are measured over a wide range of $\mathrm{RC}$ integration constants, and the results are compared with theoretical values derived in terms of the finite gain-bandwidth (GB) product and the slew rate of an op-amp and the response delay of a comparator. The comparison validates the theoretical derivation which gives the design criteria of a relaxation oscillator for capacitance measurements and sensor signal processing.
\end{abstract}

Index Terms-Capacitance measurement, comparator, operational amplifier, relaxation oscillator, signal processing.

\section{INTRODUCTION}

$\mathbf{B}$ ECAUSE of its simple configuration, a relaxation oscillator consisting of an op-amp-based integrator and a comparator is widely applied to digital capacitance measurements and conventional interfaces of capacitive transducers [1]-[6]. Applications are based on the linear relation between the oscillation period and the integration time constant $R C$ with $C$ being the capacitance under measurement. Capacitances of micromachined capacitive transducers are usually in the range of a few picofarad. Does the linear relation hold true in such a small capacitance range? The work described in this paper has been motivated by this question.

An op-amp-based integrator is also a basic constituent of active filters. According to sensitivity analysis of typical Sallen and Key filters, the sensitivity of the pole frequency $\omega_{p}$ and the pole $Q$ to the gain-bandwidth (GB) product of an op-amp increases with $\omega_{p}$, and the product $\omega_{p} Q$ of a bandpass filter is limited to one hundredth of GB if the sensitivity smaller than $1 \%$ is required [7]. This implies that the pole frequency which would otherwise be determined by the external RC time constant is affected by nonidealities of an op-amp and such a limitation would also exist in a relaxation oscillator.

To answer the question, experimentally observed oscillation period is first presented in this paper. Analysis of the oscillation period which takes nonideal performances of an op-amp and a comparator into account is then given to explain the experimental results. Finally, limitation in capacitance measurements using a relaxation oscillator is described in terms of the nonidealities.

Manuscript received September 7, 1999; revised May 24, 2000.

The author is with the Research Institute of Electronics, Shizuoka University, Hamamatsu 432-8011, Japan.

Publisher Item Identifier S 0018-9456(00)07032-7.

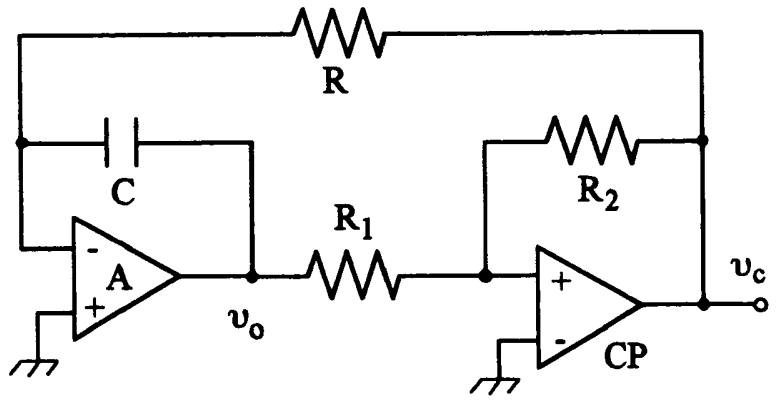

(a)

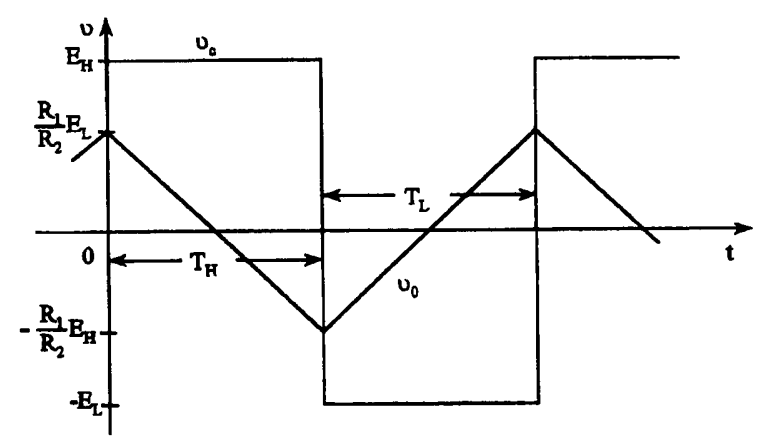

(b)

Fig. 1. (a) Circuit diagram and (b) waveforms of a relaxation oscillator.

\section{RELAXATION OSCILLATOR}

Fig. 1 shows a circuit diagram of a relaxation oscillator and waveforms observed at the outputs of the op-amp-based integrator and the comparator. Contrarily to the usual assumption, $t=0$ in Fig. 1(b) does not correspond to power turn on but to an arbitrary transition in the output voltage. Assuming for the moment ideal op-amp A and comparator CP, we have the following equation for the integrator output $v_{o}$ :

$$
C \frac{d v_{o}}{d t}+\frac{v_{c}}{R}=0
$$

where $v_{c}$ is the output voltage of the comparator which assumes $E_{H}$ until $v_{o}$ reaches $-\left(R_{1} / R_{2}\right) E_{H}$. Solving (1) under the initial condition

$$
v_{o}(t=0)=\frac{R_{1}}{R_{2}} E_{L}
$$

we obtain the expression for $T_{H}$

$$
T_{H}=C R \frac{R_{1}}{R_{2}}(1+\gamma)
$$




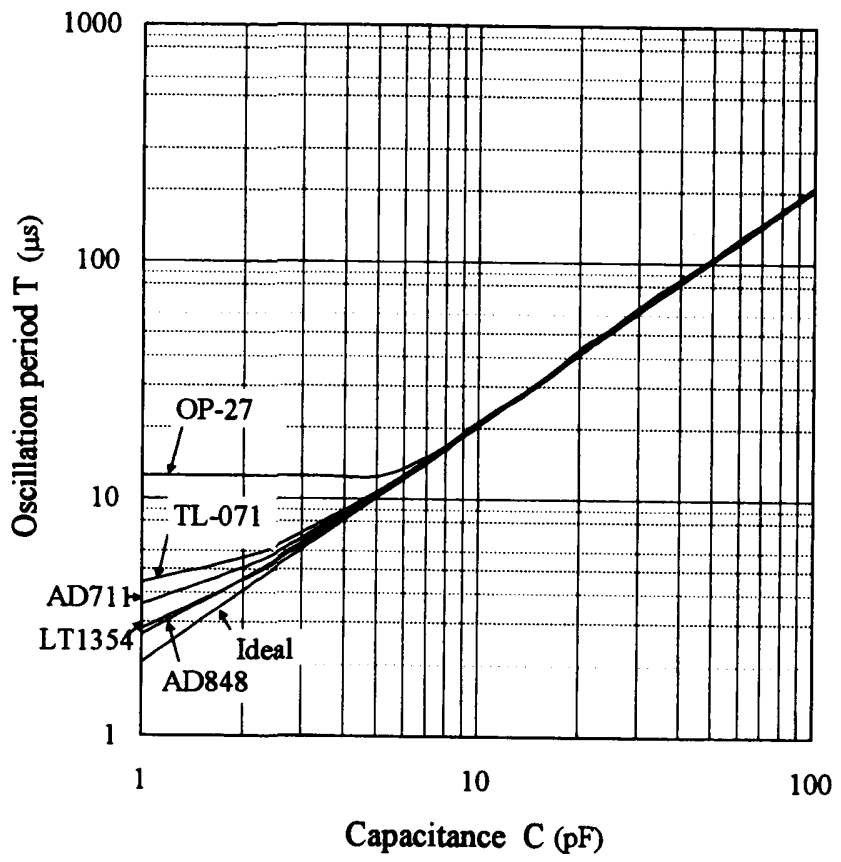

Fig. 2. Measured oscillation periods of relaxation oscillators.

where

$$
\gamma=\frac{E_{L}}{E_{H}}
$$

Similarly, the period $T_{L}$ during which the comparator assumes the negative constant voltage $-E_{L}$ is given by

$$
T_{L}=C R \frac{R_{1}}{R_{2}}\left(1+\frac{1}{\gamma}\right)
$$

The oscillation period $T$ is thus

$$
T=T_{H}+T_{L}=C R \frac{R_{1}}{R_{2}}\left(2+\gamma+\frac{1}{\gamma}\right) .
$$

Capacitance and resistance measurements using a relaxation oscillator are based on this linear relation between the oscillation period $T$ and the time constant $C R$.

The measured relations are plotted in Fig. 2. Component values and circuit parameters are: $R=1 \mathrm{M} \Omega, R_{1}=5.1 \mathrm{k} \Omega$, $R_{2}=10 \mathrm{k} \Omega, E_{H}=E_{L}=13.5 \mathrm{~V}$. Op-amps with different electrical performances were used for comparison. Taking a look at Fig. 2, one notices that the plots follow the ideal relation (6) in the capacitance range larger than $10 \mathrm{pF}$, but deviate from it in the smaller range and the deviation depends largely on the electrical performance of an op-amp.

\section{Nonideal Performance ANALYsis}

To explain the discrepancy mentioned in the previous section, we introduce nonidealities of an op-amp and a comparator into the performance analysis. In accordance with the experiment, $E_{H}=E_{L}=E$ is assumed hereafter.

Nonidealities of an op-amp are the finite GB, the slew rate, and the offset voltage. The finite GB can be described in terms of the frequency-dependent open loop gain $A(s)$

$$
A(s)=\frac{A_{o} \omega_{c}}{s+\omega_{c}} \approx \frac{A_{o}}{s}
$$

where

$A_{o} \quad$ dc gain;

$\omega_{c} \quad$ dominant pole;

$A_{o} \omega_{c} \quad$ gain-bandwidth (GB) product.

The output of the integrator in Fig. 1 when driven by the unit step $E_{H}$ is then given by

$$
V_{o}(s)=-\frac{A_{o} \omega_{c}}{\left(C+C_{i}\right) R} \cdot \frac{1}{s+\frac{1+A_{o} \omega_{c}}{\left(C+C_{i}\right) R}} \cdot \frac{E_{H}}{s^{2}}
$$

where $C_{i}$ is the input capacitance at the inverting input terminal of an op-amp. In deriving (8), the approximate expression of $A(s)$ given by (7) is used because the integrator operation at the frequency much higher than $\omega_{c}$ is analyzed. Transforming (8) into the time domain and taking the initial value (2) into account, we have

$$
\begin{aligned}
v_{o}(t)=\alpha E-\frac{E}{C R}\{ & \frac{1}{1+\frac{1}{A_{o} \omega_{c} C R}} t-\frac{1}{A_{o} \omega_{c}}\left(1+\frac{C_{i}}{C}\right) \\
& \cdot \frac{1}{\left(1+\frac{1}{A_{o} \omega_{c} C R}\right)^{2}} \\
& \left.\cdot\left(1-e^{-\left[\left(1+A_{o} \omega_{c} C R\right) /\left(C+C_{i}\right) R\right] t}\right)\right\}
\end{aligned}
$$

where

$$
\alpha=\frac{R_{1}}{R_{2}} .
$$

The first term inside the braces indicates that the finite GB product reduces the slope of the linear ramp voltage of the integrator. The second term inside the braces represents the offset voltage due to the finite GB product which invalidates the virtual-ground condition. The effect of each term inside the braces upon the oscillation period is evaluated using parameters of typical op-amps. The result shows that the effect of the last transient term is small enough to be neglected.

Similar analysis including the offset voltage $E_{o s}$ of op-amp shows that its effect on the integrator performance can be evaluated simply by shifting the input by the offset voltage. The slope $S L$ of the triangular waveform at the integrator output is limited by the slew rate, $S R$, of an op-amp. Thus, the slope is given by

$$
S L=\min \left\{\frac{E \pm E_{o s}}{C R\left(1+\frac{1}{A_{o} \omega_{c} C R}\right)}, S R\right\}
$$

where the positive sign corresponds to the negative and the minus sign to the positive ramp, respectively.

Fig. 3 shows the timing diagram of the integrator and comparator outputs when the comparator has the response delay. Referring to Fig. 3, one can obtain the expressions for $T_{H}$ and $T_{L}$

$$
T_{H}=\frac{(2 \alpha+\gamma) E}{S L_{(-)}}+\frac{\tau_{H L}+\tau_{L H}}{2}
$$




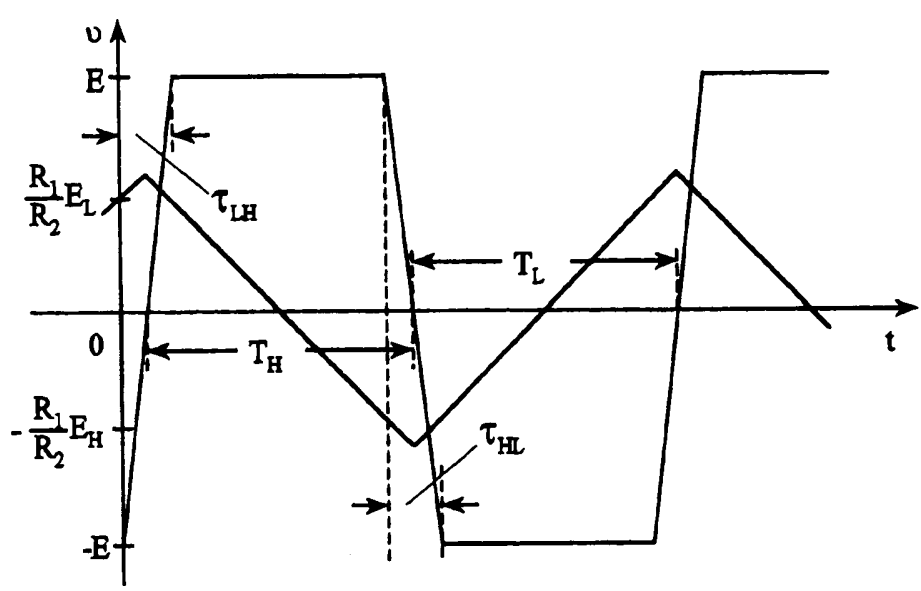

Fig. 3. Waveforms when a comparator has the response delay.

$$
T_{L}=\frac{(2 \alpha+\gamma) E}{S L_{(+)}}+\frac{\tau_{L H}+\tau_{H L}}{2}
$$

where $S L_{(-)}$and $S L_{(+)}$are the slopes of the negative and positive ramps given by (11), $\tau_{H L}$ and $\tau_{L H}$ are the response delays of a comparator at the high-to-low and low-to- high transitions, respectively, and $\gamma$ denotes the offset component due to the finite GB product in (9)

$$
\gamma=\left(1+\frac{C_{i}}{C}\right) \frac{A_{o} \omega_{c} C R}{\left(1+A_{o} \omega_{c} C R\right)^{2}} .
$$

The delay time depends on the input overdrives, and hence on the slope of ramp voltage $v_{c}$, but the dependence is neglected and $\tau_{H L}=\tau_{L H}=\tau$ is assumed to the first order approximation. The oscillation period is then given by

$$
\begin{aligned}
T= & T_{H}+T_{L} \\
= & \max \left\{(2 \alpha+\gamma) C R\left(1+\frac{1}{A_{o} \omega_{c} C R}\right)\right. \\
& \left.\cdot\left(\frac{1}{1-\Delta}+\frac{1}{1+\Delta}\right), \frac{4 \alpha E}{S R}\right\}+2 \tau \\
= & \max \left\{2(2 \alpha+\gamma) C R\left(1+\frac{1}{A_{o} \omega_{c} C R}\right), \frac{4 \alpha E}{S R}\right\}+2 \tau
\end{aligned}
$$

where

$$
\Delta=\frac{E_{o s}}{E}
$$

It is noted in (15) that the effects of the offset voltage on $T_{H}$ and $T_{L}$ cancel, to first order, each other. This also holds true of the offset voltage of a comparator.

The gain-bandwidth products $A_{o} \omega_{c}$, slew rates $S R$, and input capacitances $C_{i}$ of op-amps and the response delays of comparators used in the experiment are listed in Table I. The oscillation periods calculated by (15) using these parameter values are compared with the measured periods. Fig. 4 shows the error defined by

$$
\delta=\frac{T_{\text {calculated }}-T_{\text {measured }}}{T_{\text {calculated }}} \times 100(\%) .
$$

\begin{tabular}{|c|c|c|c|c|c|}
\hline & OP-27 & TL-071 & AD711 & LT1354 & AD848 \\
\hline 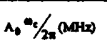 & 6 & 4 & 5 & 12 & $175^{*}$ \\
\hline $\operatorname{SR}(\mathrm{V} / \mu \mathrm{s})$ & 2.5 & 11 & 15 & $400^{*}$ & $300^{*}$ \\
\hline $\mathrm{C}_{i}(\mathrm{pF})$ & - & - & $11.0^{*}$ & $3.0^{*}$ & $1.5^{*}$ \\
\hline$\tau_{L H}(\mu s)$ & 0.53 & 0.31 & 0.32 & 0.30 & 0.30 \\
\hline$(\mu s)$ & 0.53 & 0.31 & 0.34 & 0.33 & 0.33 \\
\hline
\end{tabular}

In the capacitance range smaller than $7 \mathrm{pF}$, the oscillation period of the OP-27-based oscillator is solely determined by the
TABLE I

GAIN-BANDWIDTH PRODUCTS, SLEW RATES, AND INPUT CAPACITANCES OF OP-AMPS AND RESPONSE DELAYS OF COMPARATORS. *: MANUFACTURERS' SPECIFICATION

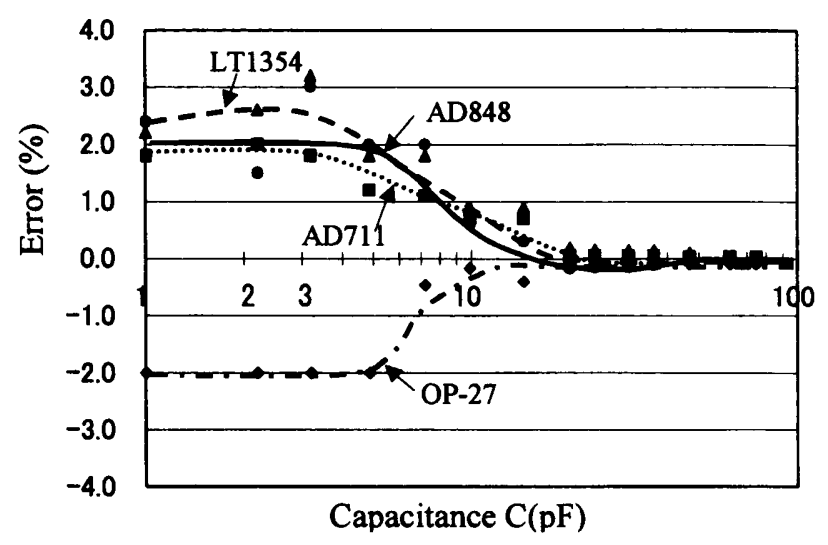

Fig. 4. Errors between the calculated and measured oscillation periods.

slew rate, and the error is independent of the capacitance. Errors for the other three op-amps are almost the same, though their GB products and input capacitances are different. This verifies the theoretical derivation given by (15) and indicates that the residual errors are largely due to the uncertainties of the delay time which depends on the input overdrives. Difficulty of an accurate characterization of a small capacitance is another source of the residual error.

\section{DESIGN CRITERIA}

The deviation of the oscillation period from the linear relation is the error in capacitance measurement

$$
\begin{aligned}
\varepsilon & =\frac{\Delta C}{C} \\
& =\frac{\Delta T}{T} \\
& =\frac{1}{A_{o} \omega_{c} C R}\left\{1+\frac{1}{2 \alpha}\left(1+\frac{C_{i}}{C}\right)\right\}+\frac{\tau}{2 \alpha C R} .
\end{aligned}
$$

The derivation of (18) assumes

$$
S R>\frac{E}{C R} .
$$

For the error to be less than $\varepsilon \%$, an op-amp and a comparator with the following specifications are required:

$$
\begin{aligned}
A_{o} \omega_{c} & >\frac{100}{\varepsilon C R}\left\{1+\frac{1}{2 \alpha}\left(1+\frac{C_{i}}{C}\right)\right\} \\
\tau & <\frac{2 \alpha \varepsilon C R}{100} .
\end{aligned}
$$


Specifically, if $1 \%$ accuracy is required in capacitance measurement of $1 \mathrm{pF}$ with $R, E$, and $\alpha$ being $1 \mathrm{M} \Omega, 10 \mathrm{~V}$, and 0.5 , respectively, then an op-amp with $A_{o} \omega_{c}$ and $S R$ larger than 72 $\mathrm{MHz}$ and $10 \mathrm{~V} / \mu$ s, respectively, and $C_{i}$ smaller than $2 \mathrm{pF}$ should be used to build a relaxation oscillator. A high-speed comparator with $\tau$ smaller than $10 \mathrm{~ns}$ is also required.

\section{CONCLUSIONS}

The effect of nonideal performances of an op-amp and a comparator upon the oscillation period of a relaxation oscillator was analyzed. The derived expression explains the nonlinear relation between the oscillation period and the integration time constant, and gives design criteria of a relaxation oscillator for capacitance measurement and sensor signal processing.

A relaxation oscillator described in this paper is also a basic building block of a voltage-to-frequency converter, charge-balancing and dual-slope analog-to-digital converters, and an oversampling $\Delta \Sigma$ modulator. The criteria derived here will also be useful for designing these data converters.

\section{REFERENCES}

[1] C. D. Johnson and H. Al Richer, "Highly accurate resistance deviation to frequency converter with programmable sensitivity and resolution," IEEE Trans. Instrum. Meas., vol. IM-35, pp. 178-181, June 1986.

[2] J. H. Huijing, G. A. van Rossum, and M. van der Lee, "Two-wire bridge-to-frequency converter," IEEE J. Solid-State Circuits, vol. SC-22, pp. 343-349, June 1987.

[3] S. S. Awad, "Capacitance measurement based on an operational amplifier circuit: Error determination and reduction," IEEE Trans. Instrum. Meas., vol. 37, pp. 379-382, Sept. 1988.

[4] F. M. L. van der Goes and G. C. M. Meijer, "A novel low-cost capacitivesensor interface," IEEE Trans. Instrum. Meas., vol. 45, pp. 536-540, Apr. 1996.

[5] K. Mochizuki and K. Watanabe, "A high-resolution, linear resistance-tofrequency converter," IEEE Trans. Instrum. Meas., vol. 45, pp. 761-764, June 1996.

[6] K. Mochizuki, K. Watanabe, T. Masuda, and M. Katsura, "A relaxation-oscillator-based interface for high-accuracy ratiometric signal processing of differential-capacitance transducers," IEEE Trans. Instrum. Meas., vol. 47, pp. 11-15, Feb. 1998.

[7] G. Daryanani, Principles of Active Network Synthesis and Design. New York: Wiley, 1976.
Yili Liu received the B.E. and M.E. degrees from Xidian University, Xi'an, China, in 1997 and 2000, respectively. He stayed at Shizuoka University, Hamamatsu, Japan, from 1998 to 1999 as an exchanging student, where he was engaged in op-amp-based analog signal processing. He is now a Design Engineer with HuaWei Institute, Beijing, China.

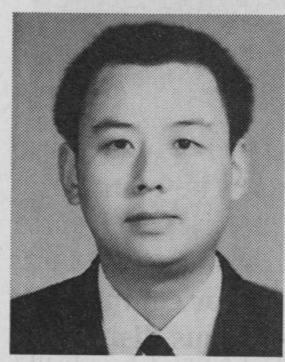

Song Chen received the B.E. degree in measurement and instrumentation and the M.E. degree in electronic engineering from Xidian University, $\mathrm{Xi}$ 'an, China, in 1994 and 1997, respectively. He is currently pursuing the $\mathrm{Ph} . \mathrm{D}$. degree under a program sponsored by the Japanese government scholarship in the Research Institute of Electronics, Shizuoka University, Hamamatsu, Japan.

He worked toward the control system design for the large- scale production lines in the 7th Design and Research Institute, Ministry of Machinery Industry, China, from 1997 to 1999. His current research interests are in the sensor signal processing and its applications.

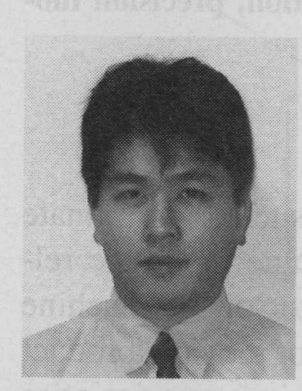

Masakatsu Nakayama graduated from Kanazawa Technical Senior High School, Kanazawa, Japan, 1990.

He joined the Research Institute of Electronics, Shizuoka University, Hamamatsu, Japan, as a technical staff member. He is currently engaged in hardware and software design and implementation of instrumentation and measurement systems.

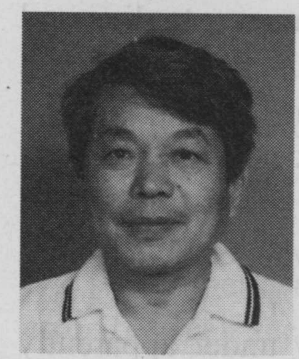

Kenzo Watanabe (M'74-SM'86-F'93) received the B.E. and M.E. degrees in engineering from Shizuoka University, Hamamatsu, Japan, in 1962 and 1966, respectively, and the Dr. Eng. degree from Kyoto University, Kyoto, Japan, in 1976.

He was with UCLA as a Visiting Professor from 1982 to 1983 . He is currently a Professor at the Research Institute of Electronics, Shizuoka University.

Dr. Watanabe is an Associate Editor of IEEE TRANSACTIONS ON INSTRUMENTATION AND MEASUREMENT. He received the Andrew R. Chi Best Paper Award and the IM Society Award from the IEEE Instrumentation and Measurement Society in 1984 and 1999, respectively, the Saito Award from the Chion Institute in 1990, and the Takayanagi Memorial Award in 1994. He was also designated an Emeritus Professor of Xidian University, Xi'an, China, in 1997. 